\title{
A family study of asymptomatic small bowel Crohn's disease
}

\author{
Livia Biancone $^{\mathrm{a}, *}$, Emma Calabrese $^{\mathrm{a}}$, Carmelina Petruzziello ${ }^{\mathrm{a}}$, Alessandra Capanna ${ }^{\mathrm{a}}$, \\ Francesca Zorzi ${ }^{a}$, Sara Onali ${ }^{a}$, Giovanna Condino $^{a}$, Elisabetta Lolli ${ }^{a}$, Cinzia Ciccacci ${ }^{b}$, \\ Paola Borgiani $^{\mathrm{b}}$, Francesco Pallone ${ }^{\mathrm{a}}$
}

a GI Unit, Department of Systems Medicine, University of Tor Vergata, Rome, Italy

${ }^{\mathrm{b}}$ Department of Biomedicine and Prevention, University of Tor Vergata, Rome, Italy

\section{A R T I C L E I N F O}

\section{Article history:}

Received 1 April 2013

Accepted 6 November 2013

Available online 17 December 2013

\section{Keywords:}

Asymptomatic CD

Crohn's disease (CD)

Family study

Small Intestine Contrast Ultrasonography (SICUS)

\begin{abstract}
A B S T R A C T
Background: Discrepancies between severity of lesions and symptoms may be observed in Crohn's disease. We prospectively assessed whether Crohn's disease may be diagnosed among asymptomatic relatives of patients, using Small Bowel Contrast Ultrasonography.

Methods: Diagnosis of asymptomatic Crohn's disease relatives was defined ultrasonographically as: bowel wall thickness $>3 \mathrm{~mm}$, bowel dilation/stricture, lumen diameter $>2.5 \mathrm{~cm}$. Diagnosis was confirmed by ileocolonoscopy. Subjects were also screened for the Leu3020insC mutation.

Results: Consent was given by 35 asymptomatic first-degree relatives of 18 Crohn's disease patients. Ultrasonography indicated increased bowel wall thickness ( $5 \mathrm{~mm}$ ) compatible with ileal Crohn's disease in 1 relative (2.8\%), a 42 year-old male. Ileocolonoscopy, histology, and radiology confirmed the diagnosis of stricturing ileal Crohn's disease. Gallbladder stones were detected in $7 / 35(20 \%)$ relatives and Leu3020insC mutation in 3/35 (8.5\%).

Conclusions: Small Bowel Contrast Ultrasonography may be a useful tool to diagnose asymptomatic small bowel Crohn's disease among first-degree relatives of patients.
\end{abstract}

(c) 2013 Editrice Gastroenterologica Italiana S.r.l. Published by Elsevier Ltd. All rights reserved.

\section{Introduction}

In Crohn's Disease (CD) discrepancies between severity of lesions and symptoms may be observed [1]; furthermore the incidence and prevalence of $\mathrm{CD}$ is higher within family groups $[1,2]$.

Small Bowel Contrast Ultrasonography (SICUS) performed by an experienced physician shows high accuracy for visualizing small bowel lesions in $\mathrm{CD}$ [3-5]; therefore we performed a prospective study to assess whether small bowel $C D$ can be diagnosed in asymptomatic CD relatives by using SICUS as a screening technique. Conventional endoscopic and radiologic analyses were then performed for relatives with SICUS findings compatible with $\mathrm{CD}$. Asymptomatic conditions associated with small bowel CD (kidney/gallbladder stones) were also assessed by ultrasound.

\footnotetext{
th Grant support: The study was supported by: Fondazione Umberto di Mario, Largo Marchiafava, 00161, Roma; PRIN 2008; Codice n. 2008X8NRH4.

* Corresponding author at: Cattedra di Gastroenterologia, Dipartimento di Medicina Interna, Università di Roma “Tor Vergata”, Via Montpellier, 1, 00133 Rome, Italy. Tel.: +3906 72596376; fax: +390620903738.

E-mail address: biancone@med.uniroma2.it (L. Biancone).
}

\section{Materials and methods}

\subsection{Study protocol}

In this prospective longitudinal study $C D$ patients referring to our tertiary IBD referral centre were asked to enrol in a family study aimed at screening for asymptomatic CD. Compliant asymptomatic relatives, including complete family groups of $\mathrm{CD}$ patients were enrolled (first degree relatives, possibly all siblings of the affected family member, and all children $>18$ years). Compliant relatives were screened for both small bowel CD, by SICUS, and for the CARD15 (Leu3020insC) mutation. In case of SICUS findings compatible with $\mathrm{CD}$, ileocolonoscopy with biopsies was performed. The presence of asymptomatic conditions associated with small bowel CD (kidney/gallbladder stones) was also assessed by ultrasound. The study was approved by the local Ethical Committee and each relative filled up a written informed consent to be tested for NOD2 mutations.

\section{2. $C D$ relatives}

Inclusion criteria were: (1) being first degree relative of a $C D$ patient in follow-up; (2) age between 18 and 75 years; no history of inflammatory bowel disease (IBD) or other gastrointestinal diseases; (3) no history of gastrointestinal symptoms or intestinal 
surgery (appendectomy allowed); (4) handing in the written informed consent. Exclusion criteria were: having performed small bowel imaging or colonoscopy during the last 5 years; severe comorbidities; obesity. At enrolment, a detailed clinical history and physical examination excluded any sign and/or symptom compatible with $\mathrm{CD}$.

\subsection{SICUS}

SICUS was performed by an experienced gastroenterologist [2-6]. Findings compatible with small bowel CD included [2,6]: (1) increased bowel wall thickness (BWT >3 mm); (2) "stiff loop"; (3) small bowel dilation (lumen diameter $>2.5 \mathrm{~cm}$ ); (4) bowel stricture (diameter $<1 \mathrm{~cm}$ ) at the level of maximally distended loop; (5) fistulae; (6) mesenteric enlargement and/or masses; (7) lymph nodes enlargement (>1 cm); (8) abscesses.

\subsection{Colonoscopy}

Ileocolonoscopy with biopsy was performed by a dedicated gastroenterologist [6] in relatives showing SICUS findings compatible with CD.

\subsection{CARD15 mutation}

Blood samples were collected from each of the compliant unaffected relatives in EDTA tubes $(5 \mathrm{ml})$. The presence of the Leu3020insC mutation was then determined [7].

\subsection{Statistical analysis}

Demographic characteristics and findings were expressed as median (range) or percentages.

\section{Results}

Fifty-five consecutive CD patients (26 males, median age 45 years, range 17-79 years) were eligible to be enrolled. Written consent to participate was given by $18 / 55$ (32\%) CD patients (13 males; median age 30 , range $17-68$; median age at diagnosis 23 ; range 16-67; $C D$ site: ileum $n=6$; neo-terminal ileum $n=9$; ileumcolon $n=3$ ). Among these $18 \mathrm{CD}$ family groups, written consent was obtained by 35 of their asymptomatic relatives. The main reasons for refusing consent were anxiety/fear to be screened for CD, or long distance from the referral centre. The grade of relationship between patients and family members included first-degree relatives ( 4 fathers, 5 mothers, 8 sons; 5 daughters, 5 brothers, 8 sisters).

SICUS detected small bowel lesions compatible with CD in 1 (2.85\%) asymptomatic relative. An increased BWT $(5 \mathrm{~mm})$ of the distal ileum (Fig. 1a) was observed with SICUS in a 42-year-old man (whose mother had fibrostricturing ileal CD). Ileocolonoscopy (Fig. 1b), histology, and small bowel follow through (Fig. 1c) confirmed the diagnosis of ileal $C D$, showing deep ulcers and a marked ulcerated stenosis of the distal ileum, with ileal dilation above stenosis. No gastrointestinal symptoms or laboratory alterations were detected. Clinical assessment performed by 3 independent dedicated gastroenterologists confirmed the absence of gastrointestinal symptoms. This CD relative showing ileal lesions appeared healthy and showed no nutritional defects (BMI 28), nor history of present or past gastrointestinal symptoms. Abdominal examination was negative. After informed discussion with the patient, mesalamine ( $2.4 \mathrm{~g} /$ day) was given, and no symptoms developed over 2 years of follow-up.

None of the remaining 34 relatives considered showed an increased BWT. After SICUS, one additional asymptomatic


Fig. 1. (Panels a-c) Images of the distal ileum in an asymptomatic 42-year-old man with familial CD (mother with fibrostricturing ileal CD), as assessed by Small Intestine Contrast Ultrasonography (SICUS) (panel a), ileocolonoscopy (panel b) and small bowel follow through (SBFT) (panel c). Panel a: SICUS shows an increased BWT ( $5 \mathrm{~mm}$; n.v. $\leq 3 \mathrm{~mm}$ ) of the distal ileum (arrow), compatible with CD. Panel $b$ : Ileocolonoscopy shows deep ulcerations surrounded by severely inflamed mucosa in the distal ileum and ileo-cecal valve, compatible with CD; Panel c: SBFT shows deep ulcers and one ulcerated stricture of the distal ileum (arrow), associated with a marked dilation above stenosis.

relative (male, age 18 years, whose father had jejuno-ileal CD) referred bloody stools (spontaneously resolved in few days). Ileocolonoscopy after bleeding detected a small rectal polyp (hyperplastic) and aphtoid ulcers in the ileum. Capsule endoscopy (SBCE) detected multiple apthoid ulcers in the small bowel and mesalazine $(2.4 \mathrm{~g} /$ day $)$ was given. No clinical or haematochemical alterations were detected. The patient appeared healthy, with no history of nonsteroidal antiinflammatory drugs (NSAIDs) use, smoking, or appendectomy. After 2 years, ileocolonoscopy with biopsies showed deep ulcerations in the distal ileum compatible with CD. 
Among the 35 asymptomatic relatives studied by ultrasound, none showed kidney stones, while 7 (20\%) showed previously undetected gallbladder stones.

\subsection{CARD15 mutation}

The Leu3020insC mutation was observed in heterozygosis in $3 / 35$ (8.5\%) relatives, all 3 showing no small bowel lesions. No Leu3020ins C mutation was detected in the 2 relatives showing CDrelated small bowel lesions. CARD15 gene mutation analysis also showed that 2/15 CD compliant patients were heterozygotes and none was homozygote for this mutation.

\section{Discussion}

In $\mathrm{CD}$, discrepancies between severity of the lesions and clinical course may be observed [1]. Early endoscopic recurrence is often asymptomatic, thus indicating that $\mathrm{CD}$ lesions may develop with no symptoms [8]. Nevertheless, severe endoscopic recurrence at 1 year is predictive of clinical relapse [9], thus supporting that early detection of asymptomatic lesions may help to prevent relapse. With this purpose, immunomodulators are indicated in inactive CD patients with severe endoscopic recurrence [10]. The increasing use of ileocolonoscopy is expanding the number of observed ileal lesions compatible with $\mathrm{CD}$ in asymptomatic subjects [11]. Findings compatible with ulcerative colitis have also been reported in asymptomatic subjects [12]. Whether these cases refer to IBD or other conditions is currently undefined.

These observations prompted us to assess, in a prospective study, whether $C D$ may be diagnosed in asymptomatic CD relatives. The recruitment rate was quite low (32\%), despite the enrolled patients being under regular follow-up. Searching for CD in asymptomatic relatives may indeed induce additional fear/anxiety present due to the $\mathrm{CD}$ diagnosis in their family member. In the present preliminary observation, ileal $C D$ was diagnosed in an adult male with no history of abdominal symptoms. The small cohort size represents the main limitation of the present preliminary observation, thus not allowing a general statement regarding the prevalence of $\mathrm{CD}$ in asymptomatic $\mathrm{CD}$ relatives. Furthermore, due to the limited number of tested relatives, the impact of NOD2 testing cannot be evaluated. Nevertheless, the ratio of asymptomatic $\mathrm{CD}$ diagnosed in our population of relatives was quite high (1/35 relatives, $2.85 \%)$. Asymptomatic CD has been reported in a higher proportion of asymptomatic CD relatives $(4 / 17 ; 23 \%)$ by using the intestinal permeability test confirmed by ileocolonoscopy [13]. Asymptomatic IBD has also been reported in few studies using clinical [14] or laboratory parameters [12].

In our study, one additional asymptomatic relative with a normal SICUS developed bloody stools after enrolment. Small bowel CD was diagnosed by using ileocolonoscopy and SBCE. As bloody stools developed after SICUS, this case was reported but was excluded from the analysis, thus considering only asymptomatic relatives. An earlier diagnosis rather than a possible asymptomatic IBD may explain the present case as well as previous findings [11-14].

The limited number of relatives tested represents the main limitation of the study. Due to the low sample size, the impact of NOD2 testing could not be evaluated. In the present study, only one of the NOD2 variants (Leu3020insC) was investigated. In our previous study in CD patients referring to the same IBD Unit [7], we indeed reported that only the Leu1007fsinsC mutation (not the Arg702Trp, Gly908Arg) appeared to be a risk factor for CD.
Superficial lesions of the ileum have also been reported in asymptomatic individuals undergoing colonoscopy for non-IBD related conditions [11]. Nevertheless, only a minority of patients (0.67\%) appear to develop CD in the long term, including patients not using NSAIDs [11]. Isolated asymptomatic ileitis has been reported to not progress to overt $C D$ in the long term, despite features of chronicity in ileal biopsies [15]. During a 2-year follow-up, gastrointestinal symptoms at time of ileocolonoscopy appeared the best predictor of progression to CD in isolated ileitis $(p<0.001)$ [15]. A careful evaluation is therefore required before considering a diagnosis of $\mathrm{CD}$ in asymptomatic individuals showing an unexpected ileitis. Potential issues when searching for asymptomatic lesions include the possible clinical implications. After detailed informed discussion with the patient, mesalamine was given also in relation to its possible chemopreventive role [10]. Whether treating asymptomatic CD patients may modify the natural history of the disease is still unknown [16]. Nevertheless, we do believe that studies screening for asymptomatic CD by using non-invasive techniques may help define the natural history of the disease.

\section{Conflict of interest}

The authors declare no conflict of interest.

\section{References}

[1] Cosnes J, Gower-Rousseau C, Seksik P, et al. Epidemiology and natural history of inflammatory bowel diseases. Gastroenterology 2011;140:1785-94.

[2] Molodecky NA, Soon IS, Rabi DM, et al. Increasing incidence and prevalence of the inflammatory bowel diseases with time, based on systematic review. Gastroenterology 2012;142:46-54

[3] Calabrese E, La Seta F, Buccellato A, et al. Crohn's Disease: a comparative prospective study of transabdominal ultrasonography, small intestine contrast ultrasonography and small bowel enema. Inflammatory Bowel Diseases 2005;11:139-45

[4] Calabrese E, Petruzziello C, Onali S, et al. Severity of postoperative recurrence in Crohn's Disease: correlation between endoscopic and sonographic findings. Inflammatory Bowel Diseases 2009;15:1635-42.

[5] Calabrese E, Zorzi F, Zuzzi S, et al. Development of a numerical index quantitating small bowel damage as detected by ultrasonography in Crohn's disease. Journal of Crohn's and Colitis 2012;6:852-60.

[6] Biancone L, Calabrese E, Petruzziello C, et al. Wireless capsule endoscopy and small intestine contrast ultrasonography in recurrence of Crohn's Disease. Inflammatory Bowel Diseases 2007;13:1256-65.

[7] Vavassori P, Borgiani P, Biancone L, et al. CARD15 mutation analysis in an Italian population:Leu1007fsinsC but neither Arg702Trp nor Gly908Arg mutations are associated with Crohn's disease. Inflammatory Bowel Diseases 2004; $10: 116-21$

[8] Rutgeerts P, Geboes K, Vantrappen G, et al. Natural history of recurrent Crohn's disease at the ileocolonic anastomosis after curative surgery. Gut 1984;25:665-72

[9] Rutgeerts P, Geboes K, Vantrappen G, et al. Predictability of the postoperative course of Crohn's Disease. Gastroenterology 1990;99:956-63.

[10] Van Assche G, Dignass A, Reinisch W, et al. The second European evidencebased consensus on the diagnosis and management of Crohn's disease: special situations. Journal of Crohn's and Colitis 2010;4:63-101.

[11] Chang HS, Lee D, Kim JC, et al. Isolated terminal ileal ulcerations in asymptomatic individuals: natural course and clinical significance. Gastrointestinal Endoscopy 2010;72:1226-32.

[12] Sakata T, Niwa Y, Goto H, et al. Asymptomatic inflammatory bowel disease with special reference to ulcerative colitis in apparently healthy persons. American Journal of Gastroenterology 2001;96:735-9.

[13] Parrilli G, Orsini L, Corsaro M, et al. Is intestinal permeability test useful for asymptomatic Crohn's disease? Inflammatory Bowel Diseases 2006;12:1189-90.

[14] Galbraith SS, Drolet BA, Kugathasan S, et al. Asymptomatic inflammatory bowel disease presenting with mucocutaneous findings. Pediatrics 2005;116: 439-44.

[15] Courville EL, Siegel CA, Vay T, et al. Isolated asymptomatic ileitis does not progress to overt Crohn's disease on long-term follow-up despite features of chronicity in ileal biopsies. American Journal of Surgical Pathology 2009;33:1341-7.

[16] Hedin CR, Stagg AJ, Whelan K, et al. Family studies in Crohn's disease: new horizons in understanding disease pathogenesis, risk and prevention. Gut 2012;61:311-8. 